\title{
Editorial
}

\section{Waveform Design for 5G and beyond Systems}

\author{
Kwonhue Choi $\mathbb{D}$
}

check for

updates

Citation: Choi, K. Waveform Design for $5 \mathrm{G}$ and beyond Systems.

Electronics 2021, 10, 2124.

https://doi.org/10.3390/

electronics10172124

Received: 6 August 2021

Accepted: 26 August 2021

Published: 1 September 2021

Publisher's Note: MDPI stays neutral with regard to jurisdictional claims in published maps and institutional affiliations.
Department of Information and Communication Engineering, Yeungnam University, Gyeongsan 712-749, Korea; gonew@yu.ac.kr

\section{Aims and Objectives}

Currently, 5G communication systems are being commercially deployed in many countries. It is also true that several new applications are expected to be realized within the pre-defined 5G capabilities. To accommodate these various new applications, 5G traffic has very diverse requirements for data rate, delay, and reliability. Such challenging requirements are met when the deployment of $5 \mathrm{G}$ is based on proper radio access technology. Consequently, multiple OFDM numerologies were adopted in the 5G NR (New Radio) standard, i.e., legacy OFDM of the $4 \mathrm{G}$ system was scaled up to cope with the requirements for enhanced mobile broadband, enhanced machine-type communications, and ultra-reliable low latency communications.

The concept of using multiple OFDM numerologies likely meets the multiple requirements for a 5G system to some extent. However, this cannot be an ultimate solution, because the traffic is radically accruing more varied characteristics and requirements when compared to the initial stage of 5G, which focused mainly on the high-speed multimedia data application. For instance, applications such as vehicular communications and robotic control require high reliability and ultra-low delay. In addition, various emerging machine-to-machine (M2M) applications have sparse traffic, with a small amount of data to be delivered. The state-of-the-art OFDM technique has some limitations when addressing the aforementioned requirements at the same time. Meanwhile, numerous waveform alternatives, such as FBMC, GFDM, and UFMC, have been explored to overcome this situation. However, they also have their pros and cons due to their intrinsic waveform properties. Hence, it is very timely to come up with modifications/variations/combinations to the waveform alternatives to OFDM, together with the state-of-the-art OFDM technique, or a new innovative waveform design for 5G systems and beyond. This Special Issue aims to provide the latest research and advances in the field of waveform design, along with the related issues of $5 \mathrm{G}$ systems and beyond.

\section{Review of the Contributions in This Issue}

As previously mentioned, this Special Issue collects the latest research and advances in the field of waveform design for 5G systems and beyond, and issues related to it. This Special Issue presents five high-quality articles on these subjects. The contents of the articles in the Special Issue are summarized below.

It is important to note that the research on waveform design needs to come with an exact performance assessment and well-balanced comparison with their competitors. In light of this objective, the first pair of articles, [1,2], provide intensive comparisons among the various waveforms used for $5 \mathrm{G}$ systems and beyond, and the employed pulse shapes, respectively. In the first article "Field Trials of SC-FDMA, FBMC, and LP-FBMC in Indoor Sub-3.5 GHz Bands" [1], a common issue in conventional methodologies for the comparison among the promising waveforms for the 5G system and beyond was addressed. These conventional comparisons all have the same critical limitations that the results were based on-a stereotyped channel model and the simple nonlinearity model of analog circuits. They are substantially different from the performance results of the waveforms in a real 
channel with a real transceiver. To address this difference, the authors have compared the performances of three waveforms, i.e., SC-FDMA, FBMC, and LP-FBMC, in a real uplink indoor channel. From these studies, they figured out that the LP-FBMC is a suitable waveform for real indoor applications. The second article, "An Overview of FIR Filter Design in Future Multicarrier Communication Systems" [2], presents a comprehensive survey on the recent advances in finite impulse response (FIR) filter design methods in multicarrier modulation (MCM)-based communication systems. First, the fundamental aspects were presented, including an introduction to existing waveform candidates and the principle of FIR filter design. Then, the methods of FIR filter design were summarized, focusing on the following three categories: frequency sampling methods, windowing-based methods, and optimization-based methods. Finally, the performances of various FIR design methods were evaluated and quantified by power spectral density (PSD) and bit error rate (BER). This paper also discusses different MCM schemes, as well as their potential prototype filters.

Furthermore, the Special Issue also addresses another aspect of waveform design, considering the fact that multi-antenna transmission has become a basic and crucial element in communication systems. A lot of work is performed on multi-antenna signal design, in conjunction with the employed waveforms. Accordingly, this Special Issues includes two articles, [3,4], which are focused on advanced signal design for space-time multi-antenna techniques for 5G systems and beyond. In the article, "Waveform Design for Space-Time Coded MIMO Systems with High Secrecy Protection" [3], the authors have presented a novel secrecy-enhanced waveform design for the quasi-orthogonal space-time block code scheme. The proposed waveform embeds dynamic artificial interference (AI) that depends on the signal, as well as on the channel gain of the legitimate receiver. The dependency of the AI on the information signal is used to reduce the difference in power allocations across the transmit antennas. In addition, the dependency of the AI on the channel gain is used to cancel the AI only at the legitimate receiver, while imposing serious interference on the passive eavesdropper. As a result, the secrecy capacity of the waveform is highly enhanced. Since the proposed method does not incur any power loss due to the addition of AI, it can be efficiently utilized in many wireless systems using STBC, resulting in diversity gain, as well as security protection. As part of this category of research, the article "Improved Pair-Wise Detections of Differential Quasi-Orthogonal Space-Time Modulation with Four Transmit Antennas" [4] proposes a new complex and real pair-wise detection for conventional differential space-time modulations, based on a quasi-orthogonal design with four transmit antennas for general QAM. Owing to the independent joint ML detection of two complex and real symbol pairs, respectively, the decoding complexity is the same as, or lower than, the conventional differential detections. The proposed detections exhibit almost identical performance to an optimum maximum-likelihood receiver, as well as an improved performance when compared with conventional pair-wise detections, especially for higher modulation order.

Last but not least, the topic of this Special Issue is multiple access (MA) capability, which is one of the most crucial measures in designing waveforms. The last article "Performance Analysis of LDS Multi Access Technique and New 5G Waveforms for V2X Communication" [5] contributes to the waveform design for 5G and beyond, concerning multiple access capability. The authors of this article have proposed new efficient MA schemes, where the low-density signature (LDS) structure is combined with two waveforms for $5 \mathrm{G}$ systems and beyond, i.e., universal filtered multi-carrier (UFMC) and filtered-OFDM waveforms. They also have simulated their proposed schemes under a vehicular channel of a vehicle to everything (V2X) communication. From this simulation, it was shown that the LDS-F-OFDM significantly achieves higher performance improvements when compared with the LDS-OFDM and LDS-UFMC in all scenarios, while maintaining an affordable complexity at the transmitter and receiver sides. These improvements are attributed to the advantages that the filtered-OFDM waveform offers through addressing the adequate filter. 
Funding: This work was partly supported by the Technology development Program of MSS [S2967489], the NRF grant funded by the Korea government (MSIT) (No. 2021R1A2C1010370), and the 2021 Yeungnam University Research Grant.

Acknowledgments: As the Guest Editor, I convey gratitude to all the authors of this Special Issue for their great contributions. All the peer reviewers who helped to evaluate the submitted manuscripts and made beneficial suggestions are also gratefully acknowledged. I also would like to thank the editorial board of Electronics, who graciously invited me to guest edit this Special Issue. Finally, I owe my special thanks to Xenia Xie at the Electronics Editorial Office. The timely completion of the Special Issue would not have been possible without her diligence.

Conflicts of Interest: The author declares no conflict of interest.

\section{References}

1. Na, D.; Jang, S.; Seo, W.-G.; Choi, K. Field Trials of SC-FDMA, FBMC and LP-FBMC in Indoor Sub-3.5 GHz Bands. Electronics 2021, 10, 573. [CrossRef]

2. Jiang, L.; Zhang, H.; Cheng, S.; Lv, H.; Li, P. An Overview of FIR Filter Design in Future Multicarrier Communication Systems. Electronics 2020, 9, 599. [CrossRef]

3. Shang, P.; Lee, H.; Kim, S. Waveform Design for Space-Time Coded MIMO Systems with High Secrecy Protection. Electronics 2020, 9, 2003. [CrossRef]

4. Kim, H.; Shang, Y.; Kim, S.; Jung, T. Improved Pair-Wise Detections of Differential Quasi-Orthogonal Space-Time Modulation with Four Transmit Antennas. Electronics 2021, 10, 1675. [CrossRef]

5. Khelouani, I.; Elbahhar, F.; Elassali, R.; Idboufker, N. Performance Analysis of LDS Multi Access Technique and New 5G Waveforms for V2X Communication. Electronics 2020, 9, 1094. [CrossRef] 